\title{
Assertive community treatment for the severely mentally ill in West Lambeth
}

\author{
Tom Craig \& Soumitra Pathare
}

The prime duty of specialist mental health services is to provide effective care to often non-compliant and unstable severely mentally ill (SMI) patients. Society expects such care to be humane and effective, but also demands that the severely ill are closely supervised to minimise risk to the public. Sadly, specialist services all too often fail on both counts. In part, this is due to the sheer complexity of care that is needed, going far beyond purely medical or therapeutic interventions. SMI patients have profound difficulties accessing and using everyday services and opportunities. Negative symptoms of apathy, self-neglect and low motivation, coupled with irrational beliefs and compounded by inadequately resourced and tightly rationed provision of social care, require mental health care services to be constantly active across areas of both medical and social provision. Services that ignore the importance of long-term, integrated care do so at their peril. A steady stream of disasters has been blamed on a lack of communication and coordination between agencies (Spokes, 1988; Ritchie et al, 1994), and follow-up studies of SMI patients managed by traditional hospital out-patient services repeatedly note a mismatch between levels of need and service uptake, with the least needy consuming the greater amount of service (Goering et al, 1984; Melzer et al, 1991).

\section{Coordinating community care: case management models}

Case management is a blanket term for a range of sirategies designed to coordinate care to ensure that SMI patients receive the treatment they need and to enable an orderly, uninterrupted movement between the diverse elements of a complex service system (Bachrach, 1981).

At one extreme of case management are simple organisational structures aimed at improving links between different providers of social and medical care. Such structures aim to ensure that patients' needs are assessed, addressed and regularly reviewed under the watchful eye of someone nominated to be in charge of the case (the 'keyworker' or 'case manager'). Case managers themselves do not necessarily provide all, or even most of the total care. In Britain, the Care Programme Approach (CPA) is a good example of this approach.

At the other extreme are models which rely on the provision of the full range of social and medical care by a single multidisciplinary team. The best known of these is the assertive community treatment (ACT) approach (Stein \& Test, 1980). This model encompasses the coordination framework of all case management systems but adds the explicit aim to provide a comprehensive range of treatment, including emergency stabilisation and longer-term rehabilitation, from within a single team. Interventions are vigorous, continuous over the long term, and typically carried out in the community, rather than at a clinic. In contrast to other case management models, ACT teams have direct control over hospital admission and discharge with a brief to minimise hospital admissions (Stein \& Test, 1980; Hoult et al, 1983; Marks et al, 1994; Essock \& Kontos, 1995).

\section{Effectiveness of case management}

Despite the rapid growth in case management, there is little evidence for its effectiveness, particularly

Tom Craig, FRCPsych, is Professor of Community Psychiatry at United Medical \& Dental Schools (Guy's \& St. Thomas'), St Thomas' Hospital, Lambeth Palace Road, London SE1 7EH. He was instrumental in establishing, and continues to work with, two Assertive Community Treatment teams in West Lambeth. Soumitra Pathare, MRCPsych, is Wellcome Research Fellow at the Academic Unit of Psychiatry at UMDS. He has worked with an ACT team in West Lambeth for the past two years. 
concerning the relative strengths of the alternatives. Four main conclusions can be drawn from reviews of this topic (Taube et al,1990; Burns \& Santos, 1995).

All models reduce the numbers of patients 'falling through the net'

In virtually all studies involving at least a minimal direct care component, case-managed patients are more likely to remain in contact with services and to be in receipt of greater medical and social care than patients managed conventionally. It seems likely that these benefits result from better coordination of care, the adoption of a 'continuous' rather than 'through-put' model of care for the SMI and the active involvement of both patients and informal carers in devising treatment programmes.

Only ACT models consistently decrease the use of in-patient care

Most ACT interventions decrease the length of stay in hospital even though the absolute rate of admission may be comparable to that in standard care. Several studies have noted that length of hospital stay returned to pre-intervention levels when the ACT team was withdrawn or when their control of discharge was blocked by hospital authorities (Test et al, 1991; Audini et al, 1994). At least one well-conducted controlled trial has found ACT to be superior to other models of intensive case management in reducing hospital utilisation (Essock \& Kontos, 1995).

Improvements in symptoms and function

These are inconsistently reported, though more often achieved by ACT than by other models of case management. Reduction in social isolation and better recreational and improved personal care are the most consistent findings (Stein \& Test, 1980; Hoult et al, 1983; Goering et al, 1988; McFarlane et al, 1992; Marks et al, 1994; McGrew et al, 1995).

\section{Greater patient satisfaction}

In most studies, patients and their relatives expressed a greater satisfaction with case management services than with standard care (Stein \& Test, 1980; Marks et al, 1994).

No study has found standard hospital care to be superior to ACT on any of these outcome measures. The evidence suggests that the best and most consistent results are obtained by approaches that employ ACT. Similarly, the least impressive results are reported by studies of purely brokerage' interventions (here the main function of the case managers is to assess the client's needs thoroughly and then purchase the necessary services from specialist providers; the case managers themselves do not take part in delivery of the clinical care), with clinical case management falling between these extremes. The handful of studies of care management and CPA in this country are uniformly disappointing, reflecting their similarity to brokerage models in which the nature of treatment is not specified and the keyworker is neither equipped to provide specialist treatments nor has any control over hospitalisation (Marshall et al, 1995; Tyrer et al, 1995).

\section{Components of an assertive community treatment service}

The constitution and work of an ACT team is governed by two principles. First, that there are effective treatments for SMI; and second, that such treatments are often inadequately delivered because of a lack of appropriate therapeutic skills within the service combined with failures in the delivery system.

The ACT approach emphasises a range of core skills delivered by a multidisciplinary team; an explicit focus on setting goals with reviewable targets for all psychological and social interventions; and the involvement of the patient and their informal carers in treatment. It is this emphasis on the therapeutic content of effective case management that dictates the unique structure of an ACT team.

\section{Essential features of the delivery system}

Case recruitment and service matching

Access to ACT is necessarily limited to the most seriously ill patients who have multiple care needs, whose illnesses are poorly controlled by standard services and who are typically non-compliant. Much of the research literature has relied on recruiting patients when they present during an acute episode (typically a relapse). However, it may not be feasible (where a radical re-structuring of a service is required and there are no additional resources) to 'double run' a new team alongside existing service provision. In such circumstances it may be preferable to begin by targeting the most needy patients who are already known to community mental health professionals and placing most emphasis on changing the activities and focus of these staff (see White et al, 1996). 
In our local services patients are identified using a staff-completed questionnaire that provides weighted scores for the number of admissions in the previous two years, a history of non-adherence or poor response to medication, a history of violence or self-harm, evidence for poor daily living skills and a paucity of social supports (scale available from the authors upon request).

In order appropriately to match the level of input to patient need, our sector service is further organised around three different levels of intensity tailored to patients' needs (Fig. 1). All three levels are provided within a single organisational structure under the overall charge of a single team leader and with one of the two sector consultants taking a lead role for the team.

Level 1 - the core ACT sub-team Each case manager in this sub-team has a case load of approximately 17 patients all of whom score in the top quarter of our screening questionnaire. These patients, comprising about a third of all those identified through screening, are the most chaotic, suffer catastrophic relapses and have a poor attachment to services with a tendency to lose contact if assertive outreach is withdrawn. The majority live alone, cope poorly with managing day-to-day and around one-quarter have comorbid problems of substance misuse. The care of these patients frequently involves daily domicillary visits to administer or supervise oral medication, to help with daily living chores or to accompany the patient to shop, pay bills and attend appointments. Compliance with medication is a major focus of the 'psychological' interventions for this group and involves close working with informal carers as well as patients, providing education about illness and the necessity for medication.

Level 2 - the outreach sub-team This deals with two groups of patients making up one-third of the cases identified. First are those who are reasonably stable in terms of mental state, housing, finances and social care needs but who tend to be unreliable in their attendance at treatment centres or require continued supervision to ensure compliance with medication. Second are patients living in private or local authority residential care who may be severely disabled but whose daily living needs are met by carers from other organisations and where the involvement of the specialist team is predominantly that of delivering health care and supporting residential care personnel. Staff in this sub-team each carry a caseload of about 30 patients. They are full members of the wider case management team. They may co-work patients who are in transition between levels and join in the provision of specialist treatments (e.g. family therapy, social skills training).

\section{Screening/referral}

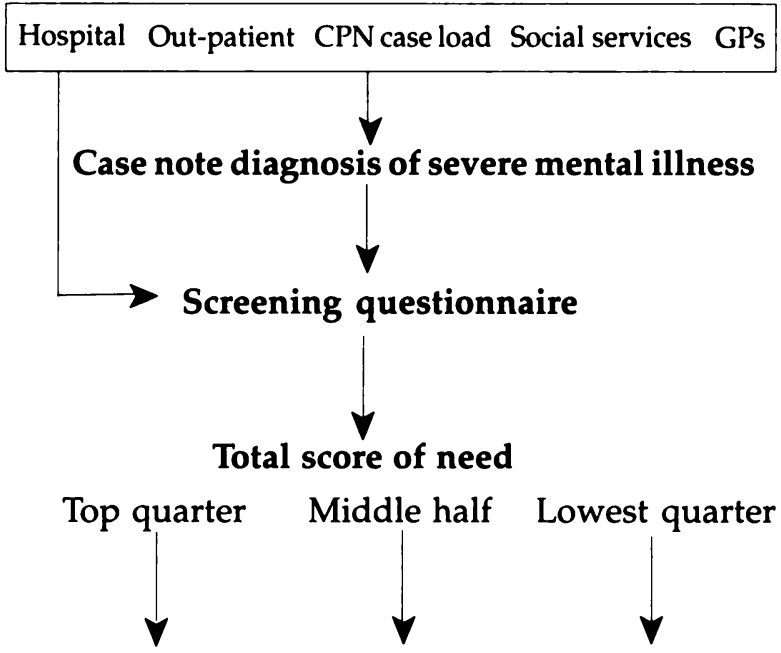

Level 1: core team Level 2: outreach Level 3: clinic

Level 3 -depot clinic The remaining patients (between a third and half of all cases known to the service) are compliant with treatment and both willing and able to attend an out-patient clinic (or community mental health centre) for treatment and supervision. Staff from both the ACT and outreach sub-teams together with sessional input from the wider mental health team join to run the outpatient clinic, which is open to patients two days a week and is the site for administering and monitoring depot neuroleptics, clozapine and lithium. The clinic includes a group-based intervention aimed at educating patients about their illness and the need for continuing medication, coping with severe mental illness and surviving in an often hostile society. Patients are transferred to this level if they have been stable for two years, if the majority of their social and health needs are being fully met and when they can be relied upon to be consistent in their attendance. If the patients' needs change or their contact with services becomes erratic, their care is moved to a higher level, as appropriate. The clinic does not accept patient referrals directly, but is seen as an in-house provision for those who are known to the team and are currently stabilised. New referrals will always spend some time with the core or outreach sub-team until the team is certain of compliance and stability.

Our service has used this multi-level model for the past four years, the advantages of which are summarised in Box 1. Our experience suggests that the majority of patients take two to three years to make the transition from level 1 to level 3 . There are, however, a minority of patients who never 
Box 1. Advantages of a three-tiered service

More efficient use of services, with input matching patient needs

Patients are known to the ACT team, which can step in quickly in case of a crisis

Easy transition of patients between levels with minimal disruption in care as they are never transferred out from the ACT team

Because of extensive co-working, the majority of patients and their carers know more than one member of the team, allowing continuity of contact even when there is a transition to a different level of care or when individual staff members are on leave or have left the service

make the transition and have to be maintained at level 1. Similarly, a significant number of patients need at least a level 2 intensity of service to be maintained effectively in the community.

\section{Team-based care}

Given the broad range of medical, psychological and social care needs of SMI patients, it is unlikely that any one professional will be able to provide the totality of care. Although patients may have a named case manager, who has prime responsibility for initial assessment and ongoing monitoring of progress, the entire team shares in providing care and supervision. In our local service this teambased approach is promoted by encouraging coworking and regular team review meetings. Coworking helps to foster a close relationship between the patient and another member of the team, which is helpful when dealing with a crisis in the absence of their own case manager. Coworking also helps to prevent staff burn-out with difficult patients by providing peer support, and can be tailored to the specific treatment needs of the patient.

One of the most common everyday problems in any large team is keeping track of transient difficulties and crises in the case-load of individual staff members. A simple innovation is the use of a large wall-board on which the names of all patients in the service are recorded on movable magnetic strips. The board is divided into colour-coded zones, red for patients who require urgent intervention to avert a specific crisis or who are relapsing, green for patients who are stable and in transition to a lower service level and amber for the remainder. The team meets briefly twice each day to review all patients in the red zone and to plan each case manager's activity for the day. The system also allows the team leader and leading psychiatrist to tell at a glance the 'temperature' of the service and to adjust staffing levels or activities accordingly. The entire team also meets for two hours each week for a detailed review of care plans for two or three patients; this meeting also provides an opportunity for team members to familiarise themselves with each others' patients and promotes cohesiveness within the team.

\section{Twenty-four-hour service provision and gatekeeping function}

A 24-hour rapid response service with the domicillary use of acute tranquillisation and intensive nursing care is an essential component of any strategy that aims to reduce hospitalisation. The control of discharge from in-patient care may well be the most important factor in reducing length of hospital stay. In the UK, other than during experimental evaluations, few community teams have provided a full 24-hour service or been in effective control over the use of hospital resources. Our local service currently operates two shifts, 12 hours daily from Monday through Saturday with a 24-hour telephone helpline out of hours. Plans are in hand to provide a full 24hour service towards the end of 1997.

\section{Assertive outreach}

An aggressive stance to the necessity for treatment is the hallmark of all ACT approaches. Even when the patient refuses interventions and help, the team continues to maintain face-to-face contact with the patient, persevering with visits despite rejection. Encouragement to accept treatment is often tied to offers of practical help with day-to-day difficulties and in some systems (notably in the USA) the links are explicit, with payment of benefits conditional on engagement with the service. If the patient refuses face-to-face contact, staff keep in touch with other informal and formal carers who are in contact with the patient, and through them maintain a constant pressure to engage in care. The use of compulsory treatment in the community, with court-imposed conditions of attendance, is common in some parts of the world and will be an increasing feature of community treatment in the UK with the introduction of supervised discharge.

\section{Multidisciplinary approach}

There is no evidence that any one group of professionals is better equipped to act as a case manager than any other. In the UK, while 
community psychiatric nurses are likely to provide the backbone to the service, the ideal team also includes a senior psychiatrist (i.e. Section 12 approved), an occupational therapist and a social worker. Sessional input from a clinical psychologist is also invaluable as a training and supervising resource. Some teams also employ unqualified health care assistants as aides to the case managers. Health care assistants do not carry an independent case load but work under the supervision of the case managers. They are involved in helping with activities of daily living, shopping, domestic chores and accompanying patients to housing and social security benefit offices. This allows the case managers to carry slightly larger case loads and to have more time for family work and other specific therapeutic interventions.

\section{Integrating the CPA}

An ACT team includes all the requirements of the CPA for the targeted patient group. The latter is the statutory framework within which the ACT provides a comprehensive needs-based service.

\section{Essential skills of an ACT team}

\section{Competency in assessment}

All professional staff in an ACT team need to be equally capable of carrying out a broad range of assessments. These include current mental state, risk of danger to self or others, and impairments of social and daily living skills. One way of ensuring generic competency is by training the team in the use of a number of semi-structured interview-based instruments. Although typically developed for use in research, many of these measures provide an excellent introduction to the difficult task of making reliable judgements of impairment, and with some thought can easily be adapted to the clinical setting.

\section{Problem-centred psychosocial interventions}

Interventions are based on an explicitly behavioural framework in which case managers and patients jointly negotiate the actions that are most likely to bring about the desired change, set mutually agreed targets for achievement and review progress regularly. Interventions may cover problems in daily living skills, self-care and domestic and occupational activities, as well as strategies to lessen the burden of abnormal mental experiences, depression and anxiety. These interventions not only ensure that patients obtain essential supplies of food, clothing and shelter during acute episodes of illness, but also help to establish routines that continue throughout the recovery phase.

\section{Medication administration and monitoring}

Interventions include the administration of depot medication, supervision of oral treatments (which might involve daily supervised administration) and the monitoring of side-effects, which are a significant factor in patients' non-compliance with medication. Structured tools for measuring the severity of side-effects are useful teaching aids and provide a framework within which wider strategies of treatment can be addressed. Compliance with medication is fostered by educational interventions and taking a negotiating stance. Patients are provided with information about the advantages and limitations of medication and encouraged to collaborate in monitoring their own treatment. Explicit interventions to enhance compliance are advocated by some teams, including 'motivational interviewing' and 'compliance groups'.

\section{Family psychoeducation and family work}

Studies have shown that family psychoeducation and therapy are effective in reducing the burden on carers, improving carers' attitudes towards the patient, improving satisfaction and reducing relapse (Leff et al, 1985). Family interventions have been reported as an explicit component of at least one ACT service (McFarlane et al, 1992) and form an integral part of our local provision.

\section{Assistance with daily living}

The role of a case manager in an ACT team includes the direct provision of social care. Team members assist patients applying for statutory benefits, help with shopping and cleaning, and advocate on their behalf in dealing with a host of statutory and nonstatutory organisations that may be involved in the total care of the patient. While the ultimate aim will be to equip patients with the skills to take care of these needs themselves, it is recognised that many will continue to require direct help indefinitely.

\section{Other skills}

There are a number of more specialised interventions that are usefully incorporated within the team's work. These include, for example, expertise in social skills training, vocational rehabilitation, cognitive therapies for treatment-resistant delusions and hallucinations, and psychological treatments of affective symptoms that often accompany severe mental illness. Such skills are usually held by only one member of the team, though the team-work approach of ACT often means that individual skills can be shared through mutual supervision. 


\section{Special populations}

The homeless The ACT approach has been successfully employed by specialist teams for homeless mentally ill people in several North American settings, achieving improved outcomes in terms of stability of re-housing, use of day care and social functioning (Morse et al, 1992; Wasylenki et al, 1993; Dixon et al, 1995).

In Britain, no strictly comparable service has been reported, though there have been a number of attempts to set up specialist services for this population (Craiget al, 1995). These are moderately successful in engaging patients but longer-term outcome is disappointing even when local systems for CPA and care management are in place and available to the specialist team (Marshall et al, 1995). This may reflect the lack of integration of homeless services with local provision, or underscore the particular difficulties of maintaining this population in long-term treatment.

Dual diagnosis ACT approaches have also been employed in the management of 'dual diagnosis' patients. Preliminary results are encouraging, with some suggestion that there may be advantages beyond those obtainable when treatments are provided by separate services for drug abuse and mental illness (Drake et al, 1990; Martin \& Scarpitti, 1993; Teague et al, 1995). To our knowledge, no comparable service exists in the UK.

\section{West Lambeth: some epidemiological and organisational features}

The catchment area of our service covers an innercity, predominantly socially deprived area in the London Borough of Lambeth. There is a high incidence of unemployment and psychiatric morbidity. The catchment area contains significant ethnic minority populations, predominantly AfroCaribbean in Brixton and Clapham (northern sector) and Asians around Streatham (southern sector). The acute and community mental health services are divided into northern and southern sectors (see Table 1). The ACT teams function out of purpose-built community mental health centres, which they share with the assessment and treatment teams for each of two sectors. The assessment and treatment teams aim to provide a short-term (up to one year) service and include nurse therapists, psychologists, behaviour therapists and part-time art therapists. Patients identified as needing long-term care are then transferred to the respective ACT teams. The ACT teams work closely with the assessment and treatment teams to provide a comprehensive service tailored to patient need.

Staff 'burnout' is frequently mentioned in the literature as a major problem with ACT teams, mainly due to the intensity of input involved and the chronicity of patient problems. In our ACT teams, there is good morale and a high degree of staff satisfaction with the model adopted, which is reflected in the low turnover of staff and the very low level of sick leave in the two teams. When asked, staff have frequently identified effective team working as the most positive aspect of their job (M. Ness, personal communication).

\section{The psychiatrist and ACT}

It is our belief that psychiatrists, with their broad training base in both pharmacological and psychotherapeutic interventions, are uniquely placed to play a leading role in the creation and maintenance of $A C T$ teams. The consultant psychiatrist provides leadership and a bridge between phases of in-patient and community care, and carries the ultimate responsibility for decisions involving compulsory hospitalisation and treatment. As the medically qualified member of the ACT team, he or she is also responsible for the drug treatments that are the mainstay of effective management of SMI. Changes in treatment require skilful supervision, particularly in the early stages, and are much easier to implement in the context of ACT that can provide the level of supervision hitherto only available to in-patients.

The place of trainees in an ACT team is less clearcut. It is generally agreed that SMI patients should be seen by the same doctor over a period of months to years. This allows the development of therapeutic alliances and collaboration in setting and achieving long-term goals. Additionally, the psychiatrist is frequently involved in situations which call for rapid decision-making around issues of safety - whether, for example, a patient can be contained by the community service or requires compulsory admission to hospital. Junior trainees rotating every six months are unable to provide the long-term continuity that is required and most are not sufficiently experienced to undertake Mental Health Act work or to take the risks that may be involved in managing a difficult case without hospitalisation. Consequently, most ACT teams rely on psychiatrists who are at least of specialist registrar grade, based full-time with the team for the main day-to-day psychiatric input. 


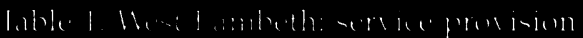

Northern sector ${ }^{1}$
Population
In-patient beds
Intensive care beds
In-patient rehabilitation beds ${ }^{2}$
ACT team started
Staff on ACT team
Level 1 case managers
Level 2 outreach nurses
Health care assistants
Medical staff ${ }^{3}$
Caseloads of individual staff
Level 1 case managers
Level 2 outreach nurses
Total team case load ${ }^{4}$

\section{0 \\ 36}

1994

12 (shared by both teams)

10 (shared by both teams)

8 (+ team leader)

4

4

2 part-time research fellows

20

30

300
Southern sector

66000

20

1992

5 (+ team leader)

3

0

1 full-time associate specialist

17

30

200

1. Soon to be divided into two smaller sectors, with separate ACT teams and a total caseload of around 150 patients.

2. In addition, there are 22 long-stay 'hospital hostel'-type beds and four respite care beds.

3. Medical staff do not carry individual caseloads and are assisted by sessional input from sector consultants.

4. Includes depot clinic patients.

\section{Conclusions}

The better results obtained with ACT-based interventions are not simply a matter of intensity of contact or small case loads. On present evidence, it appears that ACT achieves superior outcomes through the provision of a range of therapeutic interventions by a team of professionals who also have direct control of in-patient facilities.

Apart from this broad conclusion, a number of important questions remain unanswered. Which patients most need these intensive services? How long do patients have to spend under ACT supervision? How can the model be adapted to dovetail with wider community care provision? The research so far has concentrated on a fairly narrow group of patients; by and large, these studies have excluded patients whose illnesses are complicated by substance abuse or severe personality disorder or where organic aetiologies are suspected. Patients with particularly challenging behaviours involving danger to themselves or others may never be comfortably managed in community settings, and ACT may not be costeffective for patients with chronic though stable illness conditions. We also know very little about which aspects of ACT are essential for its success. For example, it may not be necessary to provide a 24-hour service if there is already a good crisis service in place. It is not at all clear whether the majority of SMI patients need to remain in ACT for many years, if not indefinitely. The multi-level model of service intensity that we have developed appears to be cost-effective and suited to our own particular local conditions. We do not know whether such a model would work in other parts of the country.

While these and other questions remain unanswered, there can be no doubt that ACT has already had a huge influence on the practice of community psychiatry.

\section{References}

Audini, B., Marks, I. M., Lawrence, R. E., et al (1994) Home-based versus out-patient/in-patient care for people with serious mental illness. Phase II of a controlled study. British Journal of Psychiatry, 165, 204-210.

Bachrach, L. L. (1981) Continuity of care for chronic mental patients: a conceptual analysis. American Journal of Psychiatry, 138, 1449-1455.

Burns, B. J. \& Santos, A. B. (1995) Assertive Community Treatment: an update of randomised trials. Psychiatric Services, 46, 669-675.

Craig, T. K. J., Bayliss, E., Klein, O., et al (1995) The Homeless Mentally Ill Initiative: An Evaluation of Four Clinical Teams. London: Department of Health.

Dixon, L. B., Krauss, N., Kernan, E., et al (1995) Modifying the PACT model to serve homeless persons with severe mental illness. Psychiatric Services, 46, 684-688.

Drake, R. E., Teague, G. B. \& Warren, R. S. (1990) New Hampshire's dual diagnosis program for people with severe mental illness and substance abuse. Addiction and Recovery, 10, 35-39.

Essock, S. M. \& Kontos, N. (1995) Implementing Assertive Community Treatment teams. Psychiatric Services, 46, 679-683. 
Goering, P., Wasylenki, D., Lancee, W., et al (1984) From hospital to community: six month and two year outcomes for 505 patients. Journal of Neroous and Mental Disease, 172, 667-673. -, Wasylenki, D. \& Farkas, M. (1988) What difference does case management make? Hospital and Community Psychiatry, 39, 272-276.

Hoult, J., Reynolds, I., Charbonneau-Powis, M., et al (1983) Psychiatric hospital versus community treatment: the results of a randomised trial. Australian and New Zealand Journal of Psychiatry, 17, 160-167.

Leff, J., Kuipers, L., Berkowitz, R., et al (1985) A controlled trial of social intervention in the families of schizophrenic patients: two year follow-up. British Journal of Psychiatry, 146, 594-600.

Marks, I. M., Connolly, J., Muijen, M., et al (1994) Home-based versus hospital-based care for people with serious mental illness. British Journal of Psychiatry, 165, 179-194.

Marshall, M., Lockwood, A. \& Gath, D. (1995) Social services case management for long-term mental disorders: a randomised controlled trial. Lancet, 345, 409-412.

Martin, S. M. \& Scarpitti, F. R. (1993) An intensive case management approach for paroled IV drug users. Journal of Drug Issues, 23, 43-59.

McFarlane, W. R., Stastny, P. \& Deakins, S. (1992) Family aided assertive community treatment: a comprehensive rehabilitation and intensive case management approach for persons with schizophrenic disorders. New Directions for Mental Health Services, 53, 43-54.

McGrew, J. H., Bond, G. R., Dietzen, L., et al (1995) A multisite study of client outcomes in assertive community treatment. Psychiatric Services, 46, 696-701.

Melzer, D., Hale, A. S., Malik, S. J., et al (1991) Community care for patients with schizophrenia one year after hospital discharge. British Medical Journal, 303, 1023-1026.

Morse, G. A., Calsyn, R. J., Allen, G., et al (1992) Experimental comparison of the effects of three treatment programs for homeless mentally ill people. Hospital and Community Psychiatry, 43, 1005-1010.

Ritchie, J. H., Dick, D. \& Lingham, R. (1994) The Report of the Inquiry into the Care and Treatment of Christopher Clunis. London: Her Majesty's Stationery Office.

Spokes, J. (1988) Report of the Committee of Inquiry into the care and aftercare of Miss Sharon Campbell (Cmd 440). London: Her Majesty's Stationery Office.

Stein, L. J. \& Test, M. A. (1980) Alternative to mental hospital treatment. 1: Conceptual model, treatment program and clinical evaluation. Archives of General Psychiatry, 37, 392397.

Taube, C. A., Morlock, L., Burns, B. J., et al (1990) New directions in research on assertive community treatment. Hospital and Community Psychiatry, 41, 642-647.

Teague, G. B., Drake, R. E. \& Ackerson, T. H. (1995) Evaluating use of continuous treatment teams for persons with mental illness and substance abuse. Psychiatric Services, 46, 689-695.

Test, M. A., Knoedler, W. H. \& Allness, D. J. (1991) Long-term community care through an assertive continuous treatment team. Advances in Neuropsychiatry, 1, 239-246.

Tyrer, P., Morgan, J. \& Van Horn, E. (1995) A randomised controlled study of close monitoring of vulnerable psychiatric patients. Lancet, 345, 756-759.

Wasylenki, D. A., Goering, P. A., Lemire, D., et al (1993) The hostel outreach program: assertive case management for homeless mentally ill persons. Hospital and Community Psychiatry, 44, 848-853.

White, K., Ness, M., Craig, T., et al (1996) Making community based comprehensive mental health services work. Psychiatric Bulletin, 20, 93-96.

\section{Multiple choice questions}

1. ACT teams reduce use of in-patient services:

a when they have control over admission and discharges

b if they follow CPA guidelines

c with a multidisciplinary approach

$d$ by reducing the length of admissions

$e$ by reducing the number of admissions.

2. In general, patients with severe mental illness:

a have significant social needs

b are not appropriate for case management

c consume the maximum amount of services

d do not respond well to assertive outreach services

e need long-term integrated care.

\section{ACT:}

a is primarily aimed at improving coordination of services

b is another term for 'case management'

c has a greater impact on symptoms and social function than clinical case management

d typically involves a multidisciplinary team

e leads to an increased carer burden.

4. The core requirements of a service aimed at people suffering from severe mental illness include:

a health care assistants to carry out activities of daily living tasks

b unfettered access to in-patient services

c a problem-centred approach to psychosocial interventions

d 24-hour availability

e individual case loads of 30 patients.

MCQ answers

$\begin{array}{lllllll}\text { 1 } & & \text { 2 } & & 3 & 4 \\ \text { a } & \text { T } & \text { a } & \text { T } & \text { a F } & \text { a F } \\ \text { b F } & \text { b F } & \text { b F } & \text { b T } \\ \text { c F } & \text { c F } & \text { c T } & \text { c T } \\ \text { d T } & \text { d F } & \text { d T } & \text { d T } \\ \text { e F } & \text { e T } & \text { e F } & \text { e F }\end{array}$

